\title{
O desempenho exercido no processo de humanização da saúde mental: uma revisão integrativa
}

\author{
The performance exercised in the humanization process of mental health: an integrative \\ review \\ El desempeño ejercido en el proceso de humanización de la salud mental: una revisión \\ integradora
}

Vanessa de Souza Correia de Araujo ${ }^{1}$, Luana Oliveira de Souza ${ }^{1}$, Karina Oliveira Duarte ${ }^{1}$, Rhuana Maria de Oliveira Pereira ${ }^{2}$, Lucas da Silva de Almeida² ${ }^{2}$ Marcelo Henrique da Silva Reis ${ }^{2 *}$, Jéssica Karoline Alves Portugal ${ }^{2}$, Sandra Jakeline Lima da Silva ${ }^{2}$, Wendel da Silva Barros², Marinelva Monteiro Dantas ${ }^{2}$.

\section{RESUMO}

Objetivo: Descrever através de uma revisão o desempenho exercido pelo CAPS no processo de humanização da saúde mental. Métodos: Trata-se de uma Revisão Integrativa de Literatura que pretendeu descrever qual o desempenho exercido pelo CAPS no processo de humanização da saúde mental. Os descritores selecionados foram: "saúde mental", "humanização" e "serviços de saúde mental". As bases de dados escolhidas para a coleta de dados foram: SCIELO, LILACS, MEDLINE e BDENF. Os critérios de inclusão foram: textos publicados nos últimos 10 anos, artigos originais, publicados em texto completo, publicados em língua portuguesa, disponibilizados gratuitamente e a amostra final foi de 4 artigos. Resultados: Os artigos selecionados versavam sobre como se dá a produção do cuidado e o uso de tecnologias de cuidado em usuários de um CAPS; uso da musicoterapia; significados atribuídos pelos usuários sobre o cuidado que lhes é prestado e compreensão dos usuários de um CAPS acerca da atenção em saúde, do ponto de vista da integralidade. Considerações finais: $O$ processo de humanização desempenhado pelo CAPS é de suma importância, pois compreende não apenas o estado clínico do indivíduo, mas também abrange os aspectos físicos, psicológicos e sociais, contribuindo sobremaneira para a humanização do portador de transtorno mental.

Palavras-chave: Saúde mental, Humanização, Serviços de saúde mental.

\begin{abstract}
Objective: To Describe through a review the performance exercised by CAPS in the humanization process of mental health. Methods: It is an Integrative Literature Review that intended to describe the performance exercised by CAPS in the humanization process of mental health. The selected descriptors were: "mental health", "humanization" and "mental health services". The databases chosen for data collection were: SCIELO, LILACS, MEDLINE and BDENF. The inclusion criteria were: texts published in the last 10 years, original articles, published in full text, published in Portuguese, made available for free and the final sample was 4 articles. Results: The selected articles dealt with how care is produced and the use of care technologies in CAPS users; use of music therapy; meanings attributed by users about the care provided to them and the understanding of users of a CAPS about health care, from the point of view of comprehensiveness. Final considerations: The humanization process performed by the CAPS is of paramount importance, as it comprises not only the individual's clinical status, but also encompasses the physical, psychological and social aspects, greatly contributing to the humanization of patients with mental disorders.
\end{abstract}

Keywords: Mental health, Humanization, Mental health services.

\footnotetext{
${ }^{1}$ Centro Universitário do Norte (UNINORTE). Manaus - AM.

*E-mail: reis.henrique.marcelo@gmail.com

2 Universidade Federal do Amazonas (UFAM). Coari - AM.
} 


\section{RESUMEN}

Objetivo: Describir a través de una revisión el desempeño de CAPS en el proceso de humanización de la salud mental. Métodos: Esta es una revisión de literatura integradora que tiene la intención de describir el desempeño ejercido por CAPS en el proceso de humanización de la salud mental. Los descriptores seleccionados fueron: "salud mental", "humanización" y "servicios de salud mental". Las bases de datos elegidas para la recolección de datos fueron: SCIELO, LILACS, MEDLINE y BDENF. Los criterios de inclusión fueron: textos publicados en los últimos 10 años, artículos originales, publicados en texto completo, publicados en portugués, disponibles gratuitamente y la muestra final fue de 4 artículos. Resultados: Los artículos seleccionados trataron sobre cómo se produce la atención y el uso de tecnologías de atención en usuarios de un CAPS; uso de musicoterapia; significados atribuidos por los usuarios sobre la atención que se les brinda y la comprensión de los usuarios de un CAPS sobre la atención médica, desde el punto de vista de la exhaustividad. Consideraciones finales: El proceso de humanización realizado por el CAPS es de suma importancia, ya que comprende no solo el estado clínico del individuo, sino que también abarca los aspectos físicos, psicológicos y sociales, contribuyendo en gran medida a la humanización de los pacientes con trastornos mentales.

Palabras clave: Salud mental, Humanización, Servicios de salud mental.

\section{INTRODUÇÃO}

Saúde Mental é um termo utilizado para retratar o grau de qualidade de vida cognitiva ou emocional do indivíduo, podendo incluir a sua capacidade de estimar a vida e buscar um equilíbrio entre as atribuições e os empenhos para atingir a plenitude psicológica. Segundo Gaino LV, et al. (2018), a saúde mental é uma condição de bem-estar no qual o indivíduo é capaz de utilizar suas próprias habilidades, restabelecer-se do estresse habitual, ser produtivo e cooperar com a sua comunidade.

A saúde mental está em processo de mudança no Brasil desde o período colonial. Raros são os dados sobre os cuidados prestados aos doentes mentais, até o século XVIII, os "loucos" eram mantidos em aposentos-prisões, nos fundos de suas casas e aqueles abandonados por suas famílias eram recolhidos para cárceres, era nítido que as diferenças sociais e econômicas tinham grande influência sobre as formas de lidar com a loucura (CARVALHO JC, 2012; SANTOS AB, et al., 2018).

A institucionalização da psiquiatria no Brasil surgiu a partir da criação das Santas Casas, onde os portadores de transtornos mentais eram agregados sem que fosse ofertado qualquer tipo de assistência. As intervenções eram realizadas em porões, onde era comum o uso de castigos físicos como forma de contenção. Na década de 90 iniciou-se o período do Movimento de Reforma Sanitária e Reforma Psiquiátrica, tendo como principal objetivo a efetivação dos cuidados às pessoas com transtorno mental, em um processo de construção de um modelo que compreendesse e respeitasse estes sujeitos como seres humanos (CARVALHO JC, 2012; SANTOS AB, et al., 2018).

Essa reforma vigorou uma nova Política de Saúde Mental, que tem como principais características: a redução de leitos e um maior controle sobre os principais hospitais psiquiátricos; a criação de rede de serviços substitutivos; a aprovação de uma nova legislação em saúde mental - a Lei n 10.216, de 06 de Abril de 2001, "que dispõe da proteção e dos direitos das pessoas portadoras de transtornos mentais, redirecionando o modelo assistencial em saúde mental, o que em última análise, influencia as questões éticas relacionadas aos cuidados prestados" (CARVALHO JC, 2012).

A principal meta da reforma psiquiátrica foi substituir a psiquiatria baseada no hospital por uma nova reforma sustentada em técnicas diversificadas, abertas e de natureza comunitária, em virtude da Luta Antimanicomial, surgindo assim, os CAPS I, CAPS II, CAPS III, CAPSi (infantil) e CAPSad (álcool e drogas), com intuito de humanizar o cuidado aos portadores de transtornos mentais (CARVALHO JC, 2012). Constituindo assim, um sistema de assistência, aplicando os princípios do Sistema Único de Saúde (SUS): universalidade, equidade e integralidade, ampliando a proposta de desinstitucionalização, do qual ultrapassa a fronteira das práticas de saúde e alcança o imaginário social e os aspectos culturalmente validados da concepção da loucura (SANTOS AB, et al., 2018). 
A humanização na saúde objetiva mudar a gestão de seus sistemas e serviços, que refere-se ao respeito pelas diferenças e especificidades de todo ser humano, caracterizando-se, como inserção das divergências nos processos de gestão e cuidados assistenciais. Essas mudanças são compostas não por uma pessoa ou grupo isolado, mas de aspecto coletivo e compartilhado, estimulando assim a produção de novas condutas do cuidar e da sistematização do trabalho (BRASIL, 2013).

Por humanização compreendemos a valorização dos diferentes sujeitos implicados no processo de produção de saúde. Os valores que norteiam essa política são três: 1: autonomia e o protagonismo dos sujeitos, 2: a co-responsabilidade entre eles e os vínculos solidários e a 3: participação coletiva no processo de gestão (BRASIL, 2007). A humanização no CAPS é decorrente de uma intrínseca relação entre os serviços de saúde, seus profissionais, o paciente e sua família, considerando as particularidades de cada contexto cultural, social e econômico (CARDOSO L, GALERA SAF, 2011).

Perante o exposto, surge algumas inquietações referente de como ocorre a humanização no CAPS, abordando com isso a seguinte pergunta norteadora: qual o desempenho exercido pelo CAPS no processo de humanização da Saúde Mental? Assim sendo, o objetivo deste trabalho é descrever, através de uma revisão integrativa da literatura, qual o desempenho exercido pelo CAPS no processo de humanização da saúde mental.

\section{MÉTODOS}

Trata-se de um estudo de Revisão Integrativa da Literatura (RIL), realizado a partir de pesquisas em artigos científicos publicados nas bases de dados Scientific Electronic Library Online (SCIELO), Literatura LatinoAmericana em Ciências da Saúde (LILACS), Literatura Internacional em Ciências da Saúde (MEDLINE) e Base de dados em Enfermagem (BDENF).

Foi realizada respeitando as 6 fases exigidas e foram utilizadas as referências teóricas para levantamento dos artigos científicos pesquisados nas bases de dados: SCIELO, LILACS MEDLINE e BDENF, utilizados os seguintes descritores: saúde mental "AND", humanização "AND" serviços de saúde mental, os quais foram extraídos do DeCs - Descritores em Ciência da Saúde. Como critérios de inclusão foram estabelecidos artigos em texto completo, em idioma português, publicados no intervalo de janeiro de 2008 a dezembro de 2018 e que correspondessem aos objetivos da revisão. Os critérios de exclusão foram: estudos do tipo teses, dissertações, monografias, revisão de literatura e textos publicados em língua estrangeira.

Para coleta de dados, previamente foram elaborados dois instrumentos semiestruturados contendo as seguintes variáveis: base de dados, revista, título, autores, objetivo, metodologia (tipo de estudo e abordagem), ano de publicação, área de interesse, formação e titulação dos autores. Comparando os dados obtidos à luz da literatura sobre o tema, realizamos a interpretação e síntese dos resultados, comparando os dados evidenciados na análise dos artigos, buscando divergências e similaridades entre os achados abordados pelos autores.

\section{RESULTADOS}

Foram identificados nas bases de dados um total de 99 artigos. Na base SCIELO foram encontradas inicialmente 4 referências, após a aplicabilidade dos filtros baseados nos critérios de inclusão e exclusão e leitura minuciosa, foi selecionado 1 artigo para compor a amostra. Na base de dados LILACS, foram obtidas 11 publicações, após a filtragem utilizando os critérios de inclusão e exclusão, restaram 10 artigos, após a leitura minuciosa restou 1 artigo para compor a amostra.

$\mathrm{Na}$ base de dados MEDLINE o resultado de pesquisa foi 0 , não havendo publicações para compor a amostra. $\mathrm{Na}$ base de dados BDENF foram levantadas 84 publicações, após a aplicabilidade dos filtros baseados no critério de inclusão e exclusão e leitura minuciosa foram selecionados 2 artigos para a composição da amostra. Assim, a amostra final utilizada para compor esta pesquisa foi de 4 artigos conforme evidenciado na Figura 1. 
Figura 1 - Fluxograma metodológico para seleção de artigos.

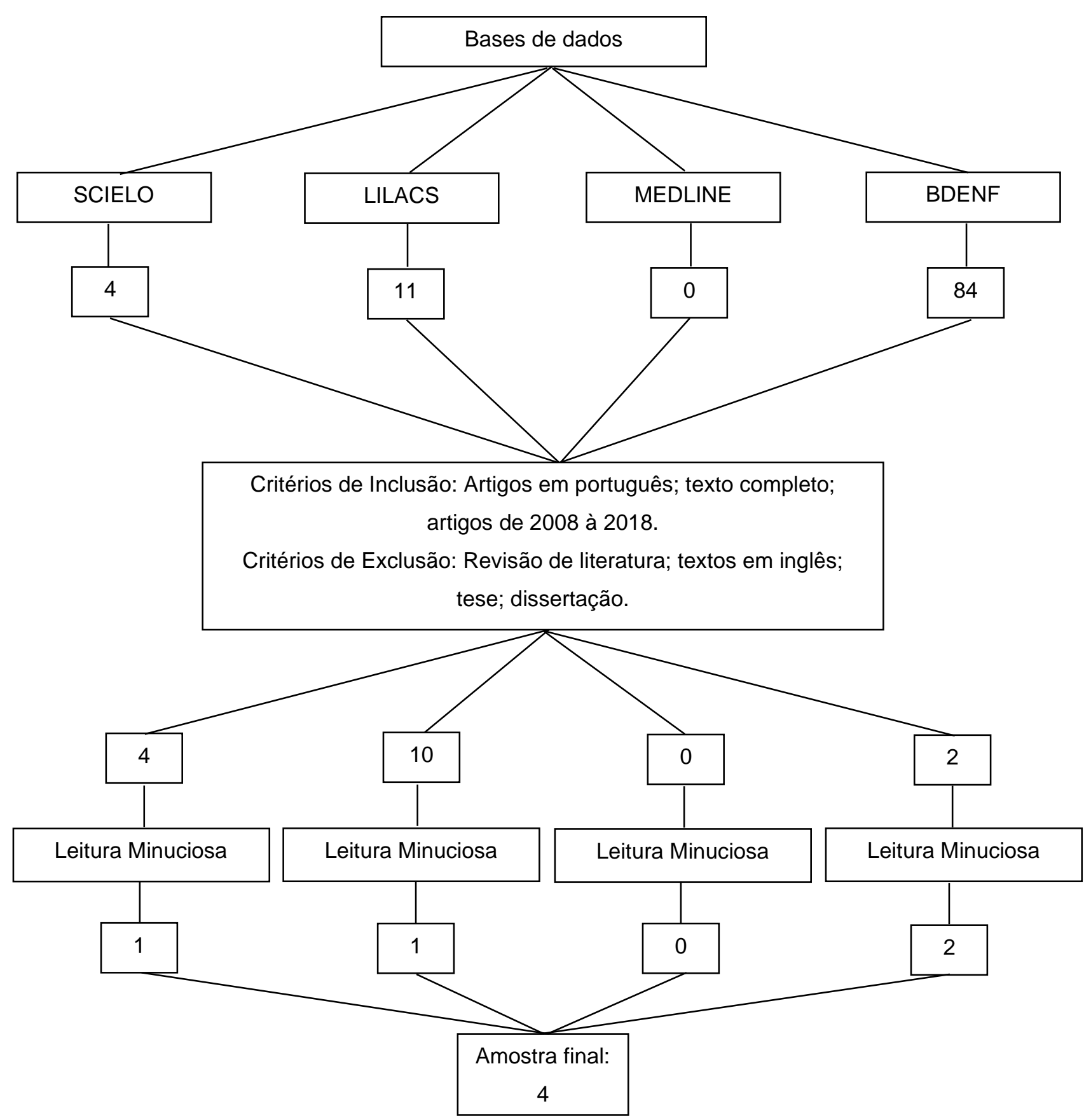

Fonte: Araujo VSC, et al., 2019.

As avaliações das variáveis Base, Revista, Titulo, Autores, Objetivo, Tipo de estudo, Abordagem e Ano estão descritas no Quadro 1, os tipos de estudos foram exploratórios, em forma de pesquisas de campo, com abordagem qualitativa, ou seja, avaliação do papel desempenhado pelo CAPS no processo de humanização da saúde mental propriamente dito e quantidades de usuários e familiares entrevistados. 
Quadro 1 - Caracterização dos estudos selecionados, segundo as bases de dados: Base, Revista, Titulo, Autores, Objetivo e Metodologia e Ano.

\begin{tabular}{|c|c|c|c|c|c|c|c|c|}
\hline \multirow{2}{*}{ № } & \multirow{2}{*}{ Base } & \multirow{2}{*}{ Revista } & \multirow{2}{*}{ Título } & \multirow{2}{*}{ Autor(es) } & \multirow{2}{*}{ Objetivo } & \multicolumn{2}{|c|}{ Metodologia } & \multirow{2}{*}{ Ano } \\
\hline & & & & & & $\begin{array}{l}\text { Tipo de } \\
\text { estudo }\end{array}$ & Abordagem & \\
\hline 1 & LILACS & $\begin{array}{l}\text { Revista de } \\
\text { Atenção } \\
\text { Primária à } \\
\text { Saúde }\end{array}$ & $\begin{array}{l}\text { Prática de Saúde Mental na } \\
\text { Rede de Atenção } \\
\text { Psicossocial: a produção do } \\
\text { cuidado e as tecnologias das } \\
\text { relações no discurso do } \\
\text { sujeito coletivo }\end{array}$ & $\begin{array}{l}\text { Barros } \\
\text { MMM, et } \\
\text { al. }\end{array}$ & $\begin{array}{l}\text { Compreender como se dá a produção do } \\
\text { cuidado e as suas tecnologias das } \\
\text { relações (humanização, acolhimento, } \\
\text { relação usuário-trabalhador de saúde) } \\
\text { dos usuários e seus respectivos } \\
\text { familiares em tratamento no Centro de } \\
\text { Atenção Psicossocial (CAPS) }\end{array}$ & Exploratório & Qualitativa & 2010 \\
\hline 2 & BDENF & $\begin{array}{l}\text { Revista } \\
\text { Enferm } \\
\text { UERJ }\end{array}$ & $\begin{array}{l}\text { Música: abrindo novas } \\
\text { fronteiras na prática } \\
\text { assistencial de enfermagem } \\
\text { em saúde mental }\end{array}$ & $\begin{array}{l}\text { Campos } \\
\text { NLe } \\
\text { Kantorski } \\
\text { LP }\end{array}$ & $\begin{array}{c}\text { Descrever a utilização da música numa } \\
\text { Oficina Terapêutica de Cuidado com o } \\
\text { Corpo. }\end{array}$ & Exploratório & Qualitativa & 2008 \\
\hline 3 & BDENF & Saúde Soc. & $\begin{array}{l}\text { Qualidade do cuidado em } \\
\text { dois centros de atenção } \\
\text { psicossocial sob o olhar de } \\
\text { usuários }\end{array}$ & $\begin{array}{l}\text { Andrade } \\
\text { AB e Bosi } \\
\text { MLM }\end{array}$ & $\begin{array}{l}\text { Compreender significados que os } \\
\text { usuários atribuem às suas experiências } \\
\text { nesses serviços e a maneira como } \\
\text { interpretam as práticas assistenciais, de } \\
\text { modo a identificar elementos que } \\
\text { favoreçam uma assistência de } \\
\text { qualidade. }\end{array}$ & Exploratório & Qualitativa & 2015 \\
\hline 4 & SCIELO & $\begin{array}{l}\text { Ciência \& } \\
\text { Saúde } \\
\text { Coletiva }\end{array}$ & $\begin{array}{l}\text { Acesso e integralidade: a } \\
\text { compreensão dos usuários } \\
\text { de uma rede de saúde mental }\end{array}$ & $\begin{array}{l}\text { Oliveira } \\
\text { RF, et al. }\end{array}$ & $\begin{array}{l}\text { Analisar as compreensões dos usuários } \\
\text { dos Centros de Atenção Psicossocial, do } \\
\text { município de Fortaleza, sobre a atenção } \\
\text { em saúde mental, com foco na } \\
\text { integralidade e no acesso. }\end{array}$ & Exploratório & Qualitativa & 2012 \\
\hline
\end{tabular}

Fonte: Araujo VSC, et al., 2019. 


\section{DISCUSSÃO}

Após a análise dos artigos selecionados, utilizamos blocos de conteúdo temáticos que conduziram os achados frequentes, como forma de descrever o papel desempenhado pelo CAPS no processo de humanização da saúde mental.

\section{A humanização no processo do cuidado no Centro de Atenção Psicossocial}

Segundo Barros MMM, et al. (2010) (Artigo 1), após a realização de um estudo em um CAPS no município de Sobral no Estado do Ceará, avaliaram que esta instituição busca realizar suas ações de forma humanística e de qualidade, utilizando os trabalhadores de saúde mental e seus gestores como o principal instrumento no processo de humanização no atendimento disponibilizado aos usuários que fazem parte da Rede de Atenção Psicossocial (RAPS), buscando considerar as pessoas em sua totalidade, pois pessoas somente são pessoas em sua inteireza.

Os usuários apontam uma assistência humanizada aos bons tratos, valorização das falas e experiências, respeito, atenção e o rompimento da postura de paciente para assumir o papel de protagonista e a infraestrutura do CAPS, que rompe com o contexto de hospital psiquiátrico, onde o cuidado ofertado se desenvolve em um ambiente que é considerado como bonito, limpo e aberto (BARROS MMM, et al., 2010).

A Política Nacional de Humanização (PNH) corrobora com o estudo de Barros uma vez que ele preconiza que o processo de humanização contempla a inclusão dos trabalhadores na gestão para que eles no seu diaa-dia reformulem seus processos de trabalho e tornem-se colaboradores que auxiliam no processo de transformação nos serviços de saúde. Além de contribuir com a inclusão de usuários e seus familiares como principal recurso no processo de cuidado, evitando assim a regressão do tratamento à pessoa com transtorno mental e ampliando a corresponsabilização no cuidar (BRASIL, 2007).

Campos NL e Kantorskil LP (2008) (Artigo 2), em seu artigo descreveram a utilização da a musicoterapia como prática do processo de humanização do cuidado ao portador de transtorno psíquico, por meio de oficinas terapêuticas, possibilitando um momento de aprendizado, humanização e crescimento interno, não apenas para os usuários, mas para os trabalhadores que dela participam.

Andrade AB e Bosi MLM (2015) (Artigo 3), avaliam a humanização através das percepções dos usuários mediante a qualidade de atendimentos oferecidos pelo Centro de Atenção Psicossocial (CAPS), pautando a importância da aceitação do usuário ao tratamento proposto pelos profissionais de saúde mental e evidenciando que o ato prescritivo deve ocorrer por meio de uma comunicação compreensível entre a equipe multiprofissional, os usuários e seus familiares. As perspectivas dos usuários apontam para a escuta, o olhar, o respeito e o diálogo, que colaboram para a adesão e continuidade do tratamento sugerido pelo CAPS.

Oliveira RF, et al. (2012) (Artigo 4), do mesmo modo avalia o processo de humanização por meio das percepções dos usuários em dez serviços de saúde mental em Fortaleza. Para os usuários a atenção de saúde mental vai além da estrutura física em si, como materiais para realização de oficinas, insuficiência de profissionais e dentre outros que compõe um centro de atenção psicossocial, porém transcende de uma atenção acolhedora, humanizada, social e espiritual onde há uma significância maior no sentimento de pertença e aceitação das singularidades dos indivíduos que do CAPS necessitam.

\section{O Centro de Atenção Psicossocial e suas tecnologias de relações implantadas como influência no cuidado em saúde mental}

Segundo Barros MMM, et al. (2010) (Artigo 1), a tecnologia é agrupada por saberes e instrumentos que apresentam relações sociais entre agentes e práticas, moldada em uma totalidade social, constituída não somente pelo saber, mas pelos desdobramentos materiais e não-materiais. As tecnologias de relações envolvidas no trabalho em saúde são classificadas em três tipos, sendo elas: leve, leve-dura e dura.

A leve refere-se à tecnologia de relação que tem como característica a produção de vínculo, acolhimento e gestão, definida nos encontros entre os diversos membros da equipe de saúde. A leve-dura refere-se aos conhecimentos estruturados que atuam no processo de trabalho em saúde, como por exemplo, a clínica médica e a epidemiologia. Já a dura concerne àquela empregada por dispositivos tecnológicos como máquinas, normas, estruturas organizacionais, entre outros (BARROS MMM, et al., 2010). 
Uma nova prática de ações comunicacionais é o acolhimento, com atos de receber e ouvir o indivíduo, promovendo respostas apropriadas a cada demanda, desde a recepção e o atendimento individual/coletivo até o encaminhamento, retorno, remarcação e alta do usuário (SCHEIBEL A e FERREIRA LH, 2012).

Campos NL e Kantorskil LP (2008), (Artigo 2) relatam os resultados das Oficinas Terapêuticas de Cuidado com o Corpo e como foi utilizada a arte musical como instrumento tecnológico para facilitar o vínculo e o diálogo, com intuito de elevar a autoestima e o autocuidado do portador de transtorno mental, estimulando o olhar, querer e cuidar de si mesmo, instigando o universo emocional e afetivo de cada usuário, tratando da individualidade e singularidade do mesmo. Além disto, descrevem a música como uma ação comunicacional que exerce uma influência incondicional sobre cada pessoa, justamente pelo fato que ela nasce da mente e das emoções do homem.

Cardoso LN, et al. (2014), afirma que independente da sua finalidade, em qualquer estado emocional do ser humano, a música tem um poder magnético de atingi-lo, atuando de forma positiva ou negativa, ou seja, resultando em evoluções como também regressões.

Scheibel A e Ferreira LH (2012), atesta que o acolhimento é alicerçado por intermédio das relações entre profissionais e usuários, caracterizando-se por meio de gestos simples como, por exemplo, a forma cordial de atendimento, referindo-se aos pacientes pelo nome, promovendo orientações em relação aos procedimentos a serem realizados de forma que esclareça suas possíveis dúvidas. Para os usuários do CAPS o acolhimento baseado na escuta e atenção é de feitio extremamente importante na qualidade do serviço em situações nas quais Ihes conferem uma certa urgência, como por exemplo em momentos de crises (JORGE MSB, et al., 2011; ARAUJO AK, et al., 2012).

Para Andrade AB e Bosi MLM (2015), o sucesso do acolhimento da crise é fundamental para o desempenho das finalidades de um CAPS, que é de assistir aos transtornos psíquicos graves e prevenir as internações. A Política Nacional de Humanização corrobora com o estudo de Andrade e Bosi uma vez que, ele preconiza que o acolhimento é distinguir que o outro traz como especificidade e distinção como necessidade de saúde, fortalecendo a relação entre profissionais e usuários (BRASIL, 2013).

O CAPS é como uma segunda casa do paciente, onde os usuários recebem assistência, mas sobretudo é um lugar em que ocorre a troca de afetos e o apoio entre eles. A maioria desses pacientes tendem a receber mais apoio humanizado no serviço de saúde mental do que em seus lares, exatamente pelo fato de se sentirem excluídos da sociedade e encontrar nesses serviços o vínculo afetivo com profissionais e outros usuários (OLIVEIRA RF, et al., 2012).

\section{O CAPS como um sistema de reinserção do portador de transtorno mental no ambiente familiar e social}

Considerando a Lei no 10.216, de 6 de abril de 2001, que dispõe sobre a proteção e os direitos das pessoas portadoras de transtornos mentais e redireciona o modelo assistencial em saúde mental, a portaria № 3.588 , de 21 de dezembro de 2017, dispõe em dois artigos a importância de:

Art. ${ }^{-} V$ - produzir, em conjunto com o usuário e seus familiares, um Projeto Terapêutico Singular que acompanhe o usuário nos contextos cotidianos, promovendo e ampliando as possibilidades de vida e mediando suas relações sociais; Art. IX - estimular o protagonismo dos usuários e familiares, promovendo atividades participativas e de controle social.

Barros MMM, et al. (2010) (Artigo 1), aborda a questão da inserção familiar no cuidado, onde é possível compreender que os familiares são personagens fundamentais na atenção às pessoas com transtornos mentais, para a obtenção de um tratamento de qualidade, resolutivo e eficiente, devido a constatação de que a terapia familiar adicionada à medicação, mostra-se mais eficaz do que a medicação em uso exclusivo. Portanto é de suma importância que os cuidadores obtenham um suporte terapêutico para lidar com as inúmeras situações que se manifestam no universo do transtorno mental, pois o mesmo acarreta danos tanto para o enfermo quanto para os seus familiares/cuidadores, transtornando o cotidiano desses últimos com surgimento de exaustão, desgaste físico e mental, transtornos do sono e apetite, revolta, temor, irritação, angústia e ansiedade (KEBBE LM, et al., 2014; GOMES MLP, et al., 2018). 
Para Barros MMM, et al. (2010) (Artigo 1), algumas ferramentas desenvolvidas na atenção às famílias são: grupos de apoio para os familiares/cuidadores, atenção domiciliar e atendimento individual. Fazendo-se imprescindível conhecer a realidade dos familiares, para assim compreender suas percepções e representações baseado em suas vivências, oferecendo-lhes apoio emocional e suporte terapêutico, proporcionando a troca de experiências, saberes e práticas, valorizando a experiência, o entendimento e os recursos empregados por eles no enfrentamento da doença.

Pelo fato de haver diversos danos para os familiares em todo o processo de cuidar do doente mental, muitos se mostram resistentes a arcar com corresponsabilização no tratamento, expressando condutas negligentes em relação aos enfermos. Diante disso o profissional deve adotar uma conduta objetiva que os levem a refletir sobre suas atitudes e as consequências das mesmas, deixando-os cientes das prováveis cobranças legais de suas ações ou omissões, porém o profissional de saúde não deve trata-los de forma arbitrária, desrespeitosa ou perversa, mas sim de forma receptiva, buscando envolvê-los no tratamento, compreendendo a dimensão do seu contexto e a história de vida de cada um, para assim se deparar com situações benéficas para o tratamento (BARROS MMM, et al., 2010).

Por conseguinte, faz-se necessário trabalhar demasiadamente com as famílias, prevenindo assim recidivas, como uma forma contribuinte na construção de uma convivência o mais saudável possível entre o indivíduo com transtorno mental e o familiar.

Andrade AB e Bosi MLM (2015) (Artigo 3) abordam a inserção social, visada na reabilitação psicossocial, tratando em facilitar espaços e novos sentidos para resgatar o protagonismo e a cidadania dos usuários, com a concepção de que a pessoa é um ser integral e deve ser acolhida por um sistema igualmente integrado, reconhecendo o valor do seu retorno ao meio social e laboral.

Algumas instituições se esforçaram para reinserir os usuários na sociedade, o CAPS3, por meio do emprego de carteira assinada em uma empresa parceira do mesmo e o CAPS5 por meio da exposição de venda de pinturas em quadro e artesanato produzidos pelos usuários, na Casa de Aprendizagem, em feiras e em alguns eventos promovidos pelo CAPS e pelo MSMCBJ.

\section{CONSIDERAÇÕES FINAIS}

Foi possível constatar que o processo de humanização ocorre através de determinadas ferramentas, como a assistência prestada pelos trabalhadores de saúde e gestores, o uso de tecnologias, como a musicoterapia inserida em oficinas terapêuticas, o acolhimento desempenhado pelo equipe de saúde mental, além disto, o CAPS atuando como um sistema de reinserção familiar, tende a tratar e facilitar espaços e novos sentidos para resgatar o protagonismo e a cidadania do portador de transtorno metal. Uma assistência humanizada é parte de um objetivo para obter maior eficiência, resultado e probabilidade de cura, quando há integração e intercomunicação entre profissionais, gestores, usuários e familiares do sistema, gerando um vínculo entre todas essas instâncias, estipulando respeito e conhecimento entre as partes envolvidas.

\section{REFERÊNCIAS}

1. ANDRADE AB, BOSI MLM. Qualidade do cuidado em dois centros de atenção psicossocial sob o olhar de usuários. Saúde e Sociedade, 2015; 24: 887-900.

2. ARAUJO AK, et al. Avaliação do processo de acolhimento em Saúde Mental na região centro-oeste do município de São Paulo: a relação entre CAPS e UBS em análise. Interface-Comunicação, Saúde, Educação, 2012; 16: $917-928$.

3. BARROS MMM, et al. Prática de saúde mental na rede de atenção psicossocial: a produção do cuidado e as tecnologias das relações no discurso do sujeito coletivo. Revista de APS, 2010; 13(1).

4. BRASIL. Ministério da Saúde. Secretaria de Atenção à Saúde. Departamento de Atenção Básica. Saúde Mental. Cadernos de Atenção Básica, n. 34. Ministério da Saúde, Secretaria de Atenção à Saúde, Departamento de Atenção Básica, Departamento de Ações Programáticas Estratégicas. Brasília: Ministério da Saúde, 2013; 176.

5. BRASIL. Ministério da Saúde. Secretaria de Atenção à Saúde. Política Nacional de Humanização. HumanizaSUS: gestão participativa: co-gestão / Ministério da Saúde, Secretaria de Atenção à Saúde, Política Nacional de Humanização. - 2. ed. rev. - Brasília: Editora do Ministério da Saúde, 2007. 
6. CAMPOS NL, KANTORSKIL LP. Música: abrindo novas fronteiras na prática assistencial de enfermagem em saúde mental. Rev. enferm. UERJ, 2008; 16(1): 88-94.

7. CARDOSO L, GALERA SAF. O cuidado em saúde mental na atualidade. Revista da Escola de Enfermagem da USP, $2011 ; 45(3)$.

8. CARDOSO LN, et al. Trocas Afetivas e Psicossociais em Musicoterapia: grupos no Centro de Atenção Psicossocial Álcool e Drogas. Revista InCantare, 2014.

9. CARVALHO JC. Diagnósticos e intervenções de enfermagem centradas no processo familiar, da pessoa com esquizofrenia. Revista Portuguesa de Enfermagem de Saúde Mental, 2012.

10. CARVALHO JC. Esquizofrenia e família: Repercussões nos filhos e cônjuge. (TESE). Universidade do Porto, 2012.

11. GAINO LV, et al. Conceito O conceito de saúde mental para profissionais de saúde. SMAD Revista Eletrônica Saúde Mental Álcool e Drogas (Edição em Português), 2018; 14(2): 108-116.

12. GOMES MLP, et al. Escutando quem cuida: quando o cuidado afeta a saúde do cuidador em saúde mental. Revista Psicologia e Saúde, 2018; 10(1).

13. JORGE MSB, et al. Promoção da Saúde Mental-Tecnologias do Cuidado: vínculo, acolhimento, co-responsabilização e autonomia. Ciência \& Saúde Coletiva, 2011; 16(7): 3051-3060.

14. KEBBE LM, et al. Cuidando do familiar com transtorno mental: desafios percebidos pelos cuidadores sobre as tarefas de cuidar. Saúde em Debate, 2014; 38(102): 494-505.

15. Lei no 10.216, de 6 de abril de 2001. Disponível em: http://planalto.gov.br/ccivil_03/leis/leis_2001/l10216.html. Acessado em: 29/02/2020.

16. OLIVEIRA RF, et al. Neusa. Acesso e integralidade: a compreensão dos usuários de uma rede de saúde mental. Ciência \& Saúde Coletiva, 2012; 17: 3069-3078.

17. Portaria no 3.588, de 21 de dezembro de 2017. Disponível em: http://bvsms.saude.gov.br/bvs/saudelegis/gm/2017/prt3588_22_12_2017.html. Acessado em: 29/02/2020.

18. SANTOS AB, et al. Saúde mental, humanização e direitos humanos. Cadernos Brasileiros de Saúde Mental/Brazilian Journal of Mental Health, 2018; 10(25): 01-19.

19. SCHEIBEL A, FERREIRA LH. Acolhimento no CAPS: reflexões acerca da assistência em saúde mental. Revista Baiana de Saúde Pública, 2012; 35(4): 966, 2012.

20. SOUZA MT, et al. Revisão integrativa: o que é e como fazer. Einstein, 2010; 8(1): 102-6. 